\title{
Nuclear Myocardial Perfusion Imaging with a Cadmium-Zinc-Telluride Detector Technique: Optimized Protocol for Scan Time Reduction
}

\author{
Bernhard A. Herzog*1, Ronny R. Buechel*1, Ruth Katz ${ }^{2}$, Michael Brueckner ${ }^{1}$, Lars Husmann ${ }^{1}$, Irene A. Burger ${ }^{1}$,
} Aju P. Pazhenkottil ${ }^{1}$, Ines Valenta ${ }^{1}$, Oliver Gaemperli ${ }^{1}$, Valerie Treyer ${ }^{1}$, and Philipp A. Kaufmann ${ }^{1,3}$

${ }^{I}$ Cardiac Imaging, University Hospital Zurich, Zurich, Switzerland; ${ }^{2}$ GE Healthcare, Tirat Hacarmel, Israel; and ${ }^{3}$ Zurich Center for Integrative Human Physiology (ZIHP), University of Zurich, Zurich, Switzerland

We aimed at establishing the optimal scan time for nuclear myocardial perfusion imaging (MPI) on an ultrafast cardiac $\gamma$-camera using a novel cadmium-zinc-telluride (CZT) solid-state detector technology. Methods: Twenty patients (17 male; BMI range,

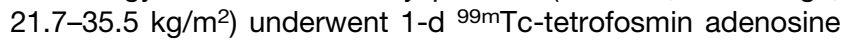
stress and rest MPI protocols, each with a 15-min acquisition on a standard dual-detector SPECT camera. All scans were immediately repeated on an ultrafast CZT camera over a 6-min acquisition time and reconstructed from list-mode raw data to obtain scan durations of $1 \mathrm{~min}, 2 \mathrm{~min}$, etc., up to a maximum of $6 \mathrm{~min}$. For each of the scan durations, the segmental tracer uptake value (percentage of maximum myocardial uptake) from the CZT camera was compared by intraclass correlation with standard SPECT camera data using a 20-segment model, and clinical agreement was assessed per coronary territory. Scan durations above which no further relevant improvement in uptake correlation was found were defined as minimal required scan times, for which Bland-Altman limits of agreement were calculated. Results: Minimal required scan times were 3 min for low dose $(r=0.81 ; P<0.001$; Bland-Altman, $-11.4 \%$ to $12.2 \%)$ and 2 min for high dose $(r=0.80 ; P<0.001$; Bland-Altman, $-7.6 \%$ to $12.9 \%$ ), yielding a clinical agreement of $95 \%$ and $97 \%$, respectively. Conclusion: We have established the minimal scan time for a CZT solid-state detector system, which allows 1-d stress/rest MPI with a substantially reduced acquisition time resulting in excellent agreement with regard to uptake and clinical findings, compared with MPI from a standard dualhead SPECT $\gamma$-camera.

Key Words: clinical cardiology; SPECT; cadmium-zinc-telluride detector; myocardial perfusion imaging; ultrafast

J Nucl Med 2010; 51:46-51

DOI: 10.2967/jnumed.109.065532

Received Apr. 27, 2009; revision accepted Sep. 25, 2009.

For correspondence or reprints contact: Philipp A. Kaufmann, Cardiac Imaging, University Hospital Zurich, Ramistrasse 100, CH-8091 Zurich, Switzerland.

E-mail: pak@usz.ch

${ }^{*}$ Contributed equally to this work.

COPYRIGHT @ 2010 by the Society of Nuclear Medicine, Inc. schemic coronary artery disease is a major cause of morbidity and mortality in industrialized countries. The hemodynamic relevance of culprit lesions can be detected and quantified noninvasively by nuclear myocardial perfusion imaging (MPI), which has grown to become the most frequently used test in nuclear medicine (1) not only for accurate diagnosis of ischemic coronary artery disease but also for assessing prognosis and for imaging myocardial viability and function (2). However, time-consuming acquisitions and cumbersome MPI protocols, with the associated costs, impaired patient comfort, and radiation exposure, have been perceived as limitations. Several attempts to improve the MPI method by using iterative reconstruction algorithms $(3,4)$, early-imaging protocols (5), or different tracers (6) provided valuable results but no breakthroughs translating into applications that improve daily clinical routine. The novel cadmium-zinc-telluride (CZT) detectors may have the potential to represent such a milestone in technical improvement of MPI. They offer a substantially improved count sensitivity as evidenced in preliminary reports (7) and first clinical studies $(8,9)$ performed on a device (D-SPECT; Spectrum Dynamics) with 9 rotating CZT detectors. An alternative approach has been developed in the current work by taking advantage of miniaturization, which allows stationary positioning of numerous CZT detectors with pinhole geometry around the heart. As a result, scans on this CZT camera (Discovery NM 530c; GE Healthcare) cover the entire heart, and the data are comparable to PET data.

The aim of this study was to establish the optimal scan time for nuclear MPI on this ultrafast cardiac $\gamma$-camera using a CZT solid-state detector.

\section{MATERIALS AND METHODS}

The present study included 20 consecutive patients who were referred for MPI because of suspected or known coronary artery 
disease. All patients underwent MPI on a standard dual-detector SPECT camera (Ventri; GE Healthcare) and on an ultrafast CZT camera immediately after the first scan. The local ethics committee approved the study protocol, and written informed consent was obtained.

\section{Study Protocol}

In all 20 patients, a $1-\mathrm{d}{ }^{99 \mathrm{~m}} \mathrm{Tc}$-tetrofosmin adenosine-stress/rest imaging protocol was performed $(10)$. The patients were told to refrain from caffeine-containing beverages for at least $12 \mathrm{~h}$ before the MPI study. Pharmacologic stress was induced by infusion of adenosine at a rate of $140 \mu \mathrm{g} / \mathrm{kg} / \mathrm{min}$. A standard dose of $300-350$ $\mathrm{MBq}$ of ${ }^{99 \mathrm{~m}} \mathrm{Tc}$-tetrofosmin was injected $3 \mathrm{~min}$ into the pharmacologic stress. Ninety minutes later, the acquisition of the stress study was performed on the Ventri, immediately followed by the acquisition on the CZT camera. Thereafter, a tracer dose 2.5-3 times the stress dose was administered at rest, followed by image acquisition on both cameras with the same protocol as at stress.

\section{SPECT MPI Image Acquisition and Reconstruction}

The first scan was acquired on a Ventri dual-head camera with a low-energy, high-resolution collimator, a $20 \%$ symmetric window at $140 \mathrm{keV}$, a $64 \times 64$ matrix, and an elliptic orbit with stepand-shoot acquisition at $3^{\circ}$ intervals over a $180^{\circ}$ arc $\left(45^{\circ}\right.$ right anterior oblique to $45^{\circ}$ left posterior oblique) with 30 steps (60 views). Scan time was set to $25 \mathrm{~s}$ per frame for stress and rest, resulting in a total acquisition time of $14 \mathrm{~min} 52 \mathrm{~s}$ (including interstep rotation time) for each scan as recommended by the American Society of Nuclear Cardiology (2). Reconstruction took place on a dedicated workstation using a standard iterative reconstruction algorithm with ordered-subset expectation maximization with 2 iterations and 10 subsets. Standard short-axis images, vertical and horizontal long-axis images, and polar maps of perfusion encompassing the entire left ventricle were produced without using resolution compensation or attenuation correction. A Butterworth postprocessing filter (frequency, 0.50; order, 10) was applied to the reconstructed slices.

\section{CZT Image Acquisition and Reconstruction}

The second scan was acquired on a CZT camera with pinhole collimation (11). In this camera, the conventional sodium iodide crystals have been replaced by CZT semiconductor technology, which directly converts radiation into electric signals without any of the steps of violet light production, transport, and conversion that occur with sodium iodide crystals (12). The energy resolution and spatial resolution (radial resolution, $4.3 \mathrm{~mm}$ ) are improved by a factor of 2 , and the sensitivity $(21.0$ counts $/ \mathrm{s} / \mathrm{mCi})$ by a factor of almost 4, compared with a dedicated cardiac $\gamma$-camera (Ventri). The CZT technology is extremely compact, and this miniaturization together with the increased resolution offers the opportunity to construct a stationary array of 19 small $\gamma$-cameras packed closely together and focused on the heart. The stationary array simultaneously acquires all the views necessary for tomographic reconstruction, saving the time required by conventional cameras for acquisitions while rotating around the subject. All views simultaneously focus on the heart to maximize the efficiency of cardiac imaging. To fit the multiple views, the image is reduced in size by means of pinhole collimation, matching the miniaturization to the improved intrinsic pixel resolution of the detectors. This allows the use of the detector surface to be maximized, increasing system efficiency. The pinhole geometry has several advantages. The reduction in pinhole sensitivity with increasing

distance significantly diminishes the contribution of background organs and tissues to the cardiac data, facilitating reliable 3-dimensional iterative reconstruction. In addition, the oblique angles of incidence also improve the already superior energy resolution of the CZT. Stress (low-dose) and rest (high-dose) scans were acquired over $6 \mathrm{~min}$, and data were saved as list file with the ability to obtain scan durations of $1 \mathrm{~min}, 2 \mathrm{~min}$, etc., up to a maximum of $6 \mathrm{~min}$. Images were reconstructed on the same workstation as for the standard SPECT acquisition using a new dedicated iterative algorithm with integrated collimator geometry modeling using maximum penalized likelihood iterative reconstruction to obtain perfusion images in standard axes. For low counts, 40 iterations have been performed, and for high counts, 50 iterations have been performed. A Butterworth postprocessing filter (frequency, 0.37; order, 7) was applied to the reconstructed slices.

\section{Image Analysis}

Image quality was graded visually on a 4-point scale as 1 (poor), 2 (fair), 3 (good), or 4 (excellent). The following parameters were considered: myocardial count density and uniformity, endocardial and epicardial edge definition, visualization, and background noise.

Quantitative analysis was performed on MPI polar maps using a 20-segment model for the left ventricle (2). Uptake was normalized to $100 \%$ peak activity, and relative percentage count uptake of $\gamma$-ray emissions was assessed for each segment from Ventri and CZT data, for each of the scan durations, and for both low and high dose.

For clinically relevant analysis, we grouped the 20 segments into the territories of the 3 main coronaries, that is, left anterior descending artery, circumflex artery, and right coronary artery, as previously suggested (2). Visual analysis was performed with regard to the presence or absence of perfusion defects in every coronary territory. Wall motion was not considered. Two experienced nuclear cardiologists made the clinical analysis by consensus.

\begin{tabular}{lc}
\hline TABLE 1. Baseline Characteristics $(n=20)$ & \\
\multicolumn{1}{c}{ Characteristic } & Value \\
Male & $17(85 \%)$ \\
Age $(\mathrm{y})$ & $66 \pm 9.4$ \\
Mean $\pm \mathrm{SD}$ & $42-80$ \\
Range & \\
BMI $\left(\mathrm{kg} / \mathrm{m}^{2}\right)$ & $27.2 \pm 3.9$ \\
Mean \pm SD & $21.7-35.5$ \\
Range & $16(80 \%)$ \\
Hypertension & $15(75 \%)$ \\
Dyslipidemia & $5(25 \%)$ \\
Diabetes & $8(40 \%)$ \\
Smoking & $1(5 \%)$ \\
Positive family history & $7(35 \%)$ \\
Previous cardiac events & $6(30 \%)$ \\
Myocardial infarction & $5(25 \%)$ \\
PCl & \\
CABG & \\
&
\end{tabular}


FIGURE 1. (A) Intraclass correlation is used to compare segmental uptake between standard $\gamma$-camera (Ventri; GE Healthcare) and ultrafast CZT camera (Discovery NM 530c; GE Healthcare). Correlation coefficient increases with prolonged scan time, but no further improvement appears after scan duration of $3 \mathrm{~min}$ is reached for low $99 \mathrm{mTC}$ tetrofosmin dose (stress scan) and 2 min for high dose (rest scan). Thus, minimally required scan times are $2 \mathrm{~min}$ for high dose and $3 \mathrm{~min}$ for low dose. (B) Diagnostic accuracy of CZT, compared with that of standard camera, increases (and significant differences according to McNemar test disappear) after scan intervals are prolonged to 3 min for low dose (stress) and $2 \mathrm{~min}$ for high dose (rest). This indicates solid clinical agreement for the above scan durations, further supported by excellent Cohen $\kappa$-value (low dose, 3 min: 0.90; high dose, 2 min: 0.92).

\section{Torso Phantom Study}

An anthropomorphic torso phantom (model ECT/TOR/P; Data Spectrum Corp.) (7) was used for the phantom studies as previously established for this type of device (13). The phantom, a large, bodyshaped cylinder, comprised the lungs, the liver, spine inserts, and cardiac inserts (model ECT/CAR/I), simulating the upper torso of average-to-large patients $(38 \times 26 \mathrm{~cm})$. The torso phantom was filled with ${ }^{99 \mathrm{~m}} \mathrm{Tc}: 57.7 \mathrm{kBq} / \mathrm{mL}(1.56 \mu \mathrm{Ci} / \mathrm{mL})$ for the cardiac insert; 38.5 $\mathrm{kBq} / \mathrm{mL}(1.04 \mu \mathrm{Ci} / \mathrm{mL})$ for the liver; $4.8 \mathrm{kBq} / \mathrm{mL}(0.13 \mu \mathrm{Ci} / \mathrm{mL})$ for low-dose background; and with $112.5 \mathrm{kBq} / \mathrm{mL}(3.04 \mu \mathrm{Ci} / \mathrm{mL})$ for the cardiac insert, $56.2 \mathrm{kBq} / \mathrm{mL}(1.52 \mu \mathrm{Ci} / \mathrm{mL})$ for the liver, and $12.6 \mathrm{kBq} / \mathrm{mL}(0.34 \mu \mathrm{Ci} / \mathrm{mL})$ for high-dose background. The heartto-liver-to-background ratio was 12:8:1 for low dose and 9:4.5:1 for high dose. Cardiac phantom studies were performed with a $2-\mathrm{cm}$ defect of $50 \%$ activity but otherwise homogeneous tracer distribution.

\section{Statistics}

SPSS software (version 15.0, SPSS Inc.) was used for statistical testing. Quantitative variables were expressed as mean $\pm \mathrm{SD}$, and categoric variables as frequencies, mean, or percentages. For each of the scan durations, the tracer uptake value (percentage of maximum myocardial uptake) from the CZT camera was compared segmentwise by intraclass correlation to Ventri data, whereas clinical agreement was assessed per coronary territory and significant differences were identified by McNemar and Cohen $\mathrm{\kappa}$-test. Scan durations above which no further improvement in relevant segmental uptake correlation was found (i.e., no more than a $5 \%$ increase in correlation coefficient) were defined as minimal required scan times. Correlation coefficient and BlandAltman limits of agreement were calculated per territories for these durations. $P$ values of less than 0.05 were considered
FIGURE 2. Linear regression analysis (left) and Bland-Altman plots (right) for per territory percentage tracer uptake at minimal required scan times; that is, 3 min for low dose (stress) (A) and 2 min for high dose (rest) (B).

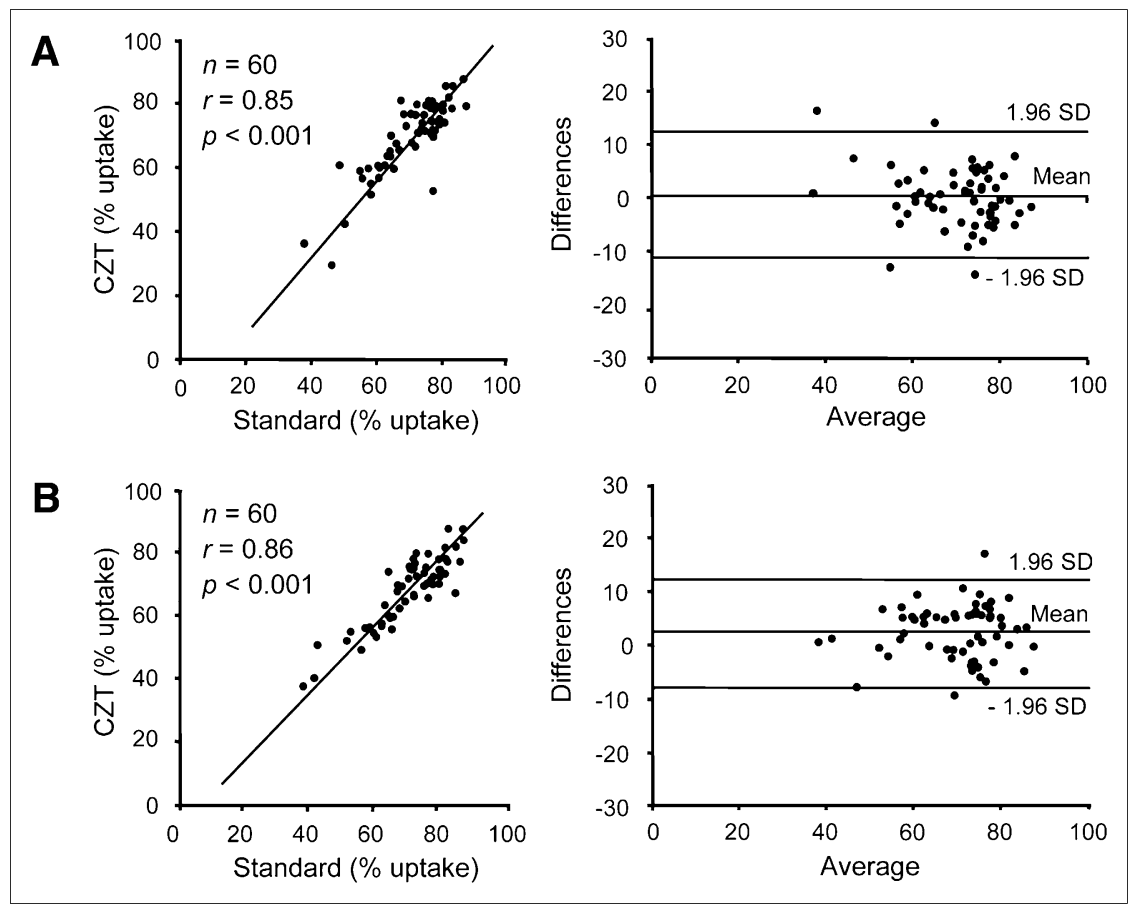


statistically significant, and the $95 \%$ confidence intervals (CIs) are presented.

\section{RESULTS}

All 20 patients successfully underwent MPI on both cameras. The baseline characteristics of the study population are given in Table 1. A mean dose of $326.8 \pm 15.7$ $\mathrm{MBq}$ of ${ }^{99 \mathrm{~m}} \mathrm{Tc}$-tetrofosmin (range, 306-362 MBq) for stress and 914.5 $\pm 12.3 \mathrm{MBq}$ (range, 894-947 MBq) for rest was administered.

\section{Image Analysis}

Image quality was graded good or excellent in all stress and rest MPI scans on the Ventri. On the CZT camera, the image quality for stress (low dose) was good or excellent in $25 \%$ of the 1 -min scans, in $80 \%$ of the 2 -min scans, and in $100 \%$ of the scans with a duration of 3 or more minutes. Comparably, for the CZT rest scans (high dose), image quality was good or excellent in $65 \%$ of the 1-min scans but reached $100 \%$ in scans with 2 or more minutes' duration.

The intraclass correlation coefficient for quantitative segmental tracer uptake of low-dose scans was 0.67 (CI, 0.45-0.76) after $1 \mathrm{~min}, 0.77(\mathrm{CI}, 0.72-0.80)$ after $2 \mathrm{~min}$, 0.81 (CI, 0.76-0.83) after 3 min, 0.79 (CI, 0.74-0.82) after $4 \mathrm{~min}, 0.82$ (CI, 0.77-0.84) after $5 \mathrm{~min}$, and 0.80 (CI, 0.750.83 ) after $6 \mathrm{~min}$. Similarly, the values for high-dose scans were 0.72 (CI, 0.60-0.80) after $1 \mathrm{~min}, 0.80$ (CI, 0.72-0.81) after $2 \mathrm{~min}, 0.81$ (CI, 0.77-0.84) after $3 \mathrm{~min}, 0.81$ (CI, 0.78-0.85) after $4 \mathrm{~min}, 0.80$ (CI, 0.78-0.85), after $5 \mathrm{~min}$, and 0.82 (CI, 0.78-0.85) after 6 min (Fig. 1). This identified 3 min (low dose) and 2 min (high dose) as minimum required scan times, because further prolongation of the scan interval did not improve correlation.

Of the 60 coronary territories $(20$ patients $\times 3$ coronary territories), 24 revealed a perfusion defect in low-dose scans and 18 in high-dose scans on the Ventri. The clinical agreement for low-dose scans was $75 \%$ (CI, 62\%-85\%) after $1 \mathrm{~min}, 87 \%$ (CI, 75\%-94\%) after 2 min, 95\% (CI, $86 \%-99 \%$ ) after 3 and $4 \mathrm{~min}$, and $97 \%$ (CI, 88\%-100\%) after 5 and $6 \mathrm{~min}$. For high-dose scans, the values were $77 \%$ (CI, 64\%-87\%) after $1 \mathrm{~min}, 97 \%$ (CI, 88\%-100\%) after 2 min, 98\% (CI, 91\%-100\%) after $3 \mathrm{~min}, 95 \%$ (CI, $86 \%-99 \%$ ) after $4 \mathrm{~min}$, and $98 \%$ (CI, 91\%-100\%) after 5 and 6 min (Fig. 1).

Bland-Altman limits of agreement for uptake in coronary territories were determined at 2 and 3 min-the minimal scan durations identified above-resulting in values of $-11.4 \%$ to $12.2 \%$ and $-7.6 \%$ to $12.9 \%$ for low- and high-dose scans, respectively (Fig. 2).

\section{Torso Phantom Study}

Figure 3 shows images of the torso phantom on the CZT and Ventri cameras at the minimal required scan times of $3 \mathrm{~min}$ for low dose and $2 \mathrm{~min}$ for high dose, demonstrating a sharper edge definition for the CZT images than for the Ventri images because of the improved spatial resolution of
FIGURE 3. Torso phantom reconstructions obtained from low- and high-dose acquisitions on CZT camera (3- and 2-min scan times, respectively) and on standard $\gamma$-camera $(15-\mathrm{min}$ scan time each) showing

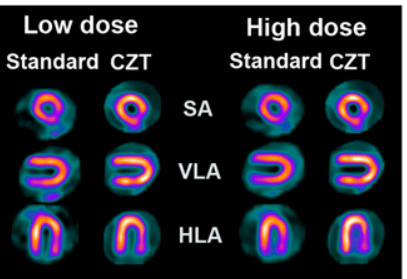
concordant defect in inferolateral wall. HLA = horizontal long axis; $\mathrm{SA}=$ short axis; VLA = vertical long axis.

the new technology. No defect was identified for either the CZT or the Ventri images. The agreement in segmental myocardial percentage uptake between CZT and Ventri is illustrated in Figure 4.

\section{DISCUSSION}

In our human study, acquisitions on a $\gamma$-camera with CZT semiconductor detectors after 1 min, 2 min, etc., up to
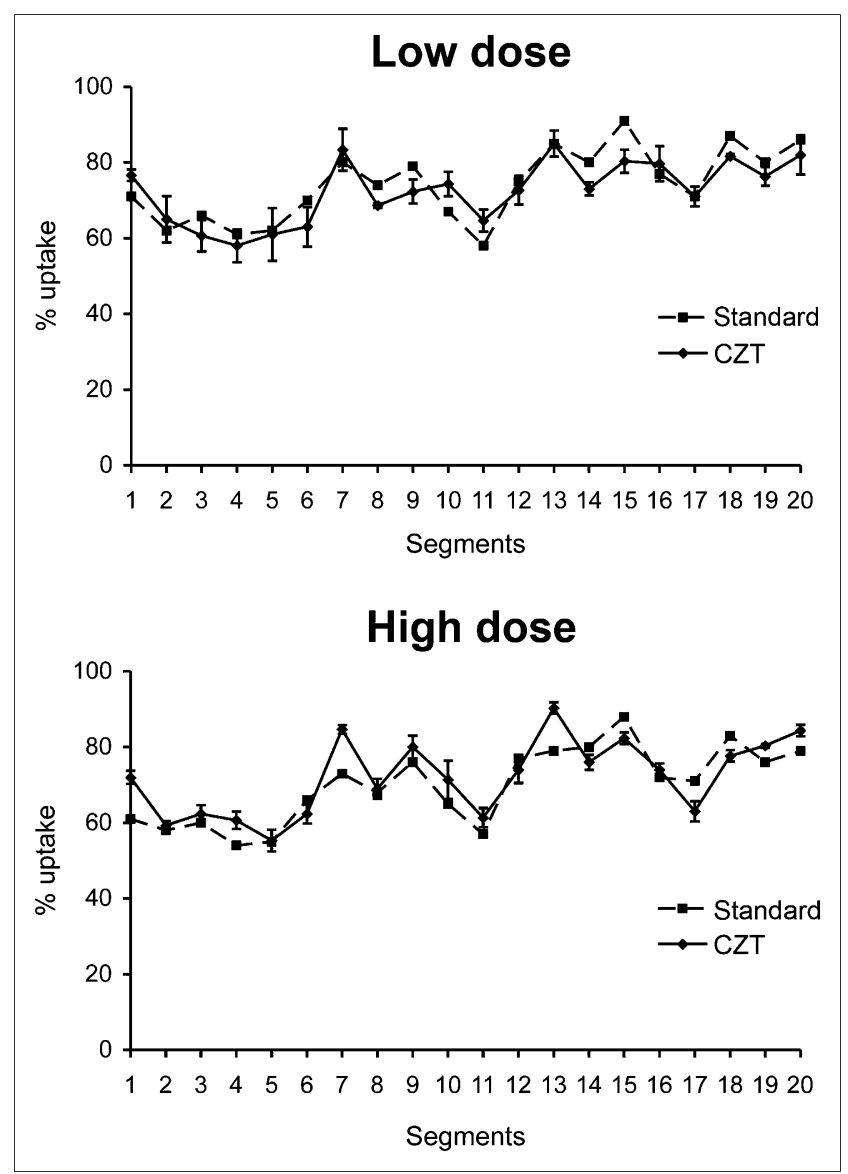

FIGURE 4. Torso phantom studies demonstrating excellent concordance of repeated measurements of percentage uptake of myocardial ${ }^{99 m T c}$ activity between standard and CZT cameras for each of 20 segments for low and high doses (respective scan times as given in Fig. 3). 
FIGURE 5. Nuclear myocardial perfusion images showing normal perfused myocardium as polar plot and short axis (SA), vertical long axis (VLA), and horizontal long axis (HLA). Images were obtained on standard $\gamma$-camera (Ventri; GE Healthcare) with acquisition time of 15 min for both stress (low dose) and rest (high dose) and on ultrafast CZT camera (Discovery NM 530c; GE Healthcare) with acquisition of $3 \mathrm{~min}$ for stress and 2 min for rest.

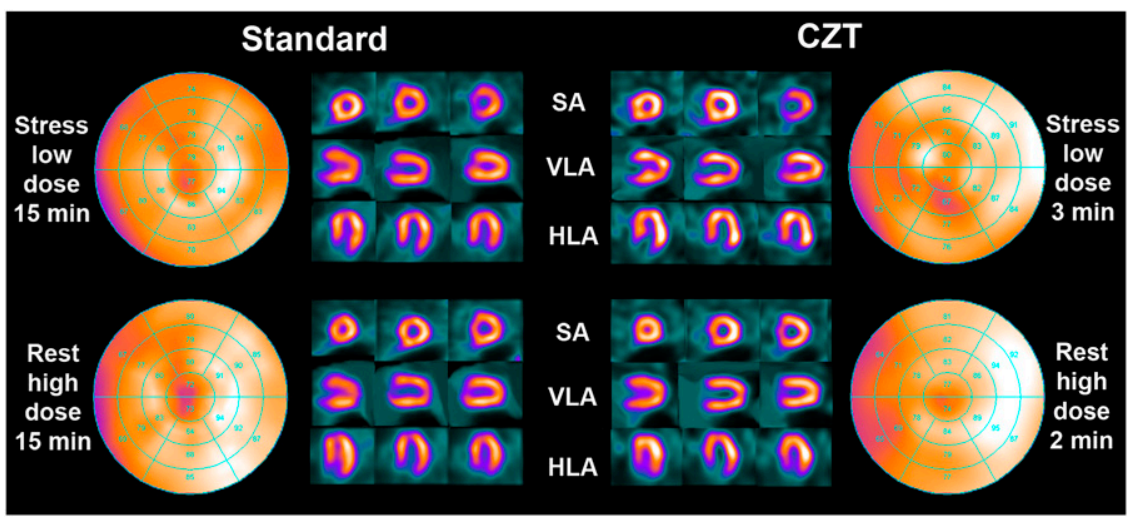

a maximum of $6 \mathrm{~min}$ for low and high ${ }^{99 \mathrm{~m}} \mathrm{Tc}$-tetrofosmin doses were compared quantitatively and qualitatively with 15 -min acquisitions on a standard dual-head $\gamma$-camera. Our results from a segmental tracer uptake comparison revealed that the CZT camera requires a minimal scan time of $3 \mathrm{~min}$ for low dose and $2 \mathrm{~min}$ for high dose to yield excellent image quality (Figs. 5 and 6) and clinical agreement with the standard camera. This finding was substantiated by the high sensitivity documented in the phantom study (Figs. 3 and 4).

In nuclear imaging, the trade-off between image acquisition time and noise levels has determined the standard protocols and scan times. Standard scan times have been successfully reduced by the introduction of multihead $\gamma$-cameras in the past decade, allowing simultaneous acquisition of multiple projections. Other attempts to shorten protocol time by allowing noisier images (14) or reduced spatial resolution (15) did not result in clinically acceptable image quality. The recently introduced iterative reconstruction algorithms incorporate noise regularization and resolution recovery and may provide a new reconstruction alternative enabling half-time acquisition while maintaining diagnostic accuracy (16).

The CZT camera, with stationary semiconductor technology offering increased resolution and minimized scan time, may replace conventional SPECT cameras and therefore may represent a major breakthrough for daily use of MPI in clinical routine. The clinical impact of shorter acquisitions lies not only in improved patient comfort and consequently fewer motion artifacts but also in increased patient throughput and consequently greater scanner efficiency. As an alternative to shortened acquisition time, the new technology may be used to reduce tracer activity and consequently effective radiation exposure to patients and nuclear staff. Furthermore, this alternative would make MPI more suitable for noninvasive hybrid cardiac imaging in combination with CT coronary angiography (17-19). The combination of MPI and low-dose CT using prospective electrocardiography triggering (20-22) would allow hybrid images to be obtained at a low total effective radiation dose (23).

The shortened scan durations established in the present study compare well with those recently obtained on a different type of CZT camera with 9 mobile parallel-hole detectors (7-9). By contrast, the present scanner consists of 19 stationary detectors located around the patient to cover the whole heart. Pinhole magnification was historically used to achieve high resolution but was hampered by a loss in system efficiency. The introduction of CZT detectors allows the advantages of pinhole imaging without a tradeoff in resolution efficiency. Furthermore, because the detector geometry with complete coverage of the entire heart at any point in time allows the acquisition of time-activity curves similar to those acquired with PET, a potential exists for myocardial blood flow quantification.

The following issues may be seen as limitations of this study. First, we did not correct for attenuation, as the aim of this study was primarily to establish the optimal scan time
FIGURE 6. Nuclear myocardial perfusion images showing perfusion defect of anteroapical myocardium, with clinical agreement between standard camera (15-min acquisition) and CZT camera (3- and 2-min acquisitions).

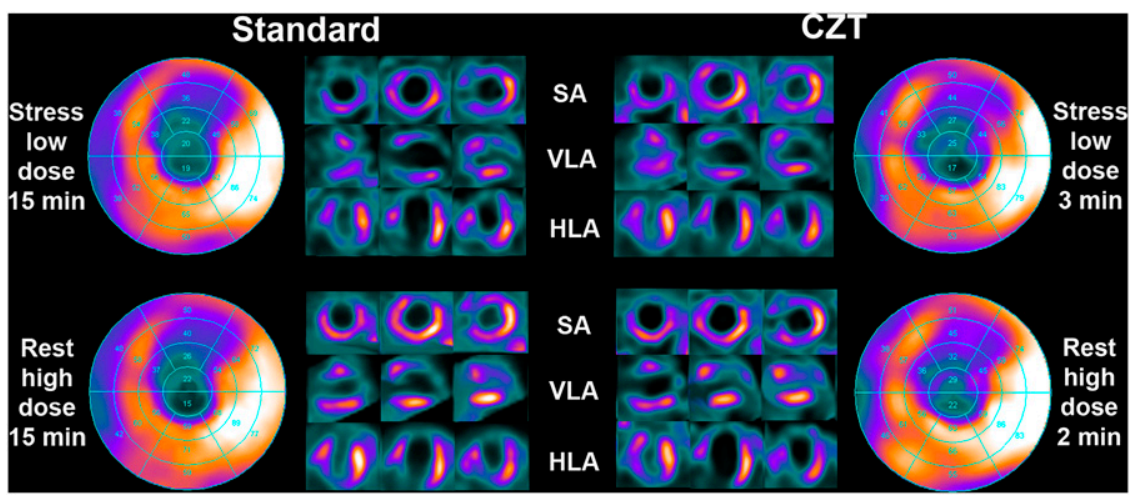


and not to show the feasibility of attenuation correction on the CZT camera. Second, our patient population consisted of only 20 patients because of the pilot nature of this study, which aimed at establishing minimal scan duration. Therefore, larger prospective trials will be needed to provide clinical validation, which was beyond the scope of the present study. Third, we did not assess scan durations between full-minute intervals. Although this choice was arbitrary, we believe that full-minute scan durations would prove most suitable for reasons of practicability. Furthermore, we included patients with a body mass index ranging from 21.7 to $35.5 \mathrm{~kg} / \mathrm{m}^{2}$. Therefore, caution must be taken when extrapolating our data to patients with a substantially higher body mass index. Finally, the leveling off of the segmental concordance at 0.8 suggests that differences remained between the 2 sets of images. Although CZT images were always acquired after the Ventri images, the delay was kept minimal, and it is therefore unlikely that this unrandomized sequence may account for the differences. Future trials may establish which reconstruction parameters could be further optimized to improve concordance.

\section{CONCLUSION}

We have established the minimal scan time for a CZT solid-state detector system that allows 1-d stress/rest MPI with a substantially reduced acquisition time. Compared with a standard dual-head SPECT $\gamma$-camera, the new system showed excellent agreement with regard to uptake and clinical findings.

\section{ACKNOWLEDGMENTS}

The study was supported by a grant from the Swiss National Science Foundation (SNSF-professorship grant) and by the ZIHP (Zurich Center for Integrative Human Physiology, University of Zurich, Switzerland). We are grateful to Josephine Trinckauf, Ennio Mueller, Mirjam De Bloeme, Verena Weichselbaumer, and Edlira Loga for their excellent technical support.

\section{REFERENCES}

1. Thomas GS, Miyamoto MI, Morello AP III, et al. Technetium $99 \mathrm{~m}$ sestamibi myocardial perfusion imaging predicts clinical outcome in the community outpatient setting. The Nuclear Utility in the Community (NUC) Study. J Am Coll Cardiol. 2004;43:213-223.

2. Hansen CL, Goldstein RA, Akinboboye OO, et al. Myocardial perfusion and function: single photon emission computed tomography. J Nucl Cardiol. 2007;14:e39-e60.
3. Borges-Neto S, Pagnanelli RA, Shaw LK, et al. Clinical results of a novel wide beam reconstruction method for shortening scan time of Tc-99m cardiac SPECT perfusion studies. J Nucl Cardiol. 2007;14:555-565.

4. DePuey EG, Gadiraju R, Clark J, Thompson L, Anstett F, Shwartz SC. Ordered subset expectation maximization and wide beam reconstruction "half-time" gated myocardial perfusion SPECT functional imaging: a comparison to "fulltime" filtered backprojection. J Nucl Cardiol. 2008;15:547-563.

5. Giorgetti A, Rossi M, Stanislao M, et al. Feasibility and diagnostic accuracy of a gated SPECT early-imaging protocol: a multicenter study of the Myoview Imaging Optimization Group. J Nucl Med. 2007;48:1670-1675.

6. Kapur A, Latus KA, Davies G, et al. A comparison of three radionuclide myocardial perfusion tracers in clinical practice: the ROBUST study. Eur J Nucl Med Mol Imaging. 2002;29:1608-1616.

7. Gambhir SS, Berman DS, Ziffer J, et al. A novel high-sensitivity rapidacquisition single-photon cardiac imaging camera. J Nucl Med. 2009;50:635-643.

8. Sharir T, Ben-Haim S, Merzon K, Prochorov V, Dickman D, Berman DS. Highspeed myocardial perfusion imaging initial clinical comparison with conventional dual detector anger camera imaging. JACC Cardiovasc Imaging. 2008; 1:156-163.

9. Berman DS, Kang X, Tamarappoo B, et al. Stress thallium-201/rest technetium$99 \mathrm{~m}$ sequential dual isotope high-speed myocardial perfusion imaging. JACC Cardiovasc Imaging. 2009;2:273-282.

10. Hesse B, Tagil K, Cuocolo A, et al. EANM/ESC procedural guidelines for myocardial perfusion imaging in nuclear cardiology. Eur J Nucl Med Mol Imaging. 2005;32:855-897.

11. Blevis I, Tsukerman L, Volokh L, Hugg J, Jansen F, Bouhnik J. CZT gamma camera with pinhole collimator: spectral measurements. IEEE Nucl Sci Symp Conf Rec. 2008:4931-4932.

12. Madsen MT. Recent advances in SPECT imaging. J Nucl Med. 2007;48:661673.

13. Volokh L, Lahat C, Binyamin E, Blevis I. Myocardial perfusion imaging with an ultra-fast cardiac SPECT camera: a phantom study. IEEE Nucl Sci Symp Conf Rec. 2008:4636-4639.

14. Taillefer R, Primeau M, Costi P, Lambert R, Leveille J, Latour Y. Technetium$99 \mathrm{~m}$-sestamibi myocardial perfusion imaging in detection of coronary artery disease: comparison between initial (1-hour) and delayed (3-hour) postexercise images. J Nucl Med. 1991;32:1961-1965.

15. DePuey EG, Nichols KJ, Slowikowski JS, et al. Fast stress and rest acquisitions for technetium-99m-sestamibi separate-day SPECT. J Nucl Med. 1995;36:569574.

16. Ali I, Ruddy TD, Almgrahi A, Anstett FG, Wells RG. Half-time SPECT myocardial perfusion imaging with attenuation correction. J Nucl Med. 2009; 50:554-562.

17. Gaemperli O, Schepis T, Kalff V, et al. Validation of a new cardiac image fusion software for three-dimensional integration of myocardial perfusion SPECT and stand-alone 64-slice CT angiography. Eur J Nucl Med Mol Imaging. 2007;34:1097-1106.

18. Gaemperli O, Schepis T, Valenta I, et al. Cardiac image fusion from stand-alone SPECT and CT: clinical experience. J Nucl Med. 2007;48:696-703.

19. Namdar M, Hany TF, Koepfli P, et al. Integrated PET/CT for the assessment of coronary artery disease: a feasibility study. J Nucl Med. 2005;46:930-935.

20. Herzog BA, Husmann L, Burkhard N, et al. Accuracy of low-dose computed tomography coronary angiography using prospective electrocardiogramtriggering: first clinical experience. Eur Heart J. 2008;29:3037-3042.

21. Husmann L, Herzog BA, Gaemperli O, et al. Diagnostic accuracy of computed tomography coronary angiography and evaluation of stress-only single-photon emission computed tomography/computed tomography hybrid imaging: comparison of prospective electrocardiogram-triggering vs. retrospective gating. Eur Heart J. 2009;30:600-607.

22. Husmann L, Valenta I, Gaemperli O, et al. Feasibility of low-dose coronary CT angiography: first experience with prospective ECG-gating. Eur Heart J. 2008;29:191-197.

23. Herzog BA, Husmann L, Landmesser U, Kaufmann PA. Low-dose computed tomography coronary angiography and myocardial perfusion imaging: cardiac hybrid imaging below 3mSv. Eur Heart J. 2009;30:644. 